Acta Crystallographica Section A

Foundations of Crystallography

ISSN 0108-7673

Received 26 June 2008

Accepted 20 November 2008

C 2009 International Union of Crystallography

Printed in Singapore - all rights reserved

\section{Comments on tables of magnetic space groups}

\author{
Hans Grimmer
}

\author{
Laboratory for Developments and Methods, Condensed Matter Research with Neutrons and Muons, \\ Paul Scherrer Institut, CH-5232 Villigen PSI, Switzerland. Correspondence e-mail: \\ hans.grimmer@psi.ch
}

Litvin [Acta Cryst. (2008), A64, 419-424 and supplementary material] extends much of the information contained in Volume A of International Tables for Crystallography for the 230 space-group types to the 1651 types of Shubnikov space groups, using Opechowski-Guccione (OG) notation for the space groups with a black-white lattice. It is pointed out that $O G$ notation has crucial disadvantages compared to Belov-Neronova-Smirnova (BNS) notation. It is shown how Litvin's diagrams of symmetry elements for the orthorhombic Shubnikov space groups can be interpreted in terms of BNS symbols and how those containing $e$-glides can be simplified. A number of mistakes in the diagrams of Litvin are corrected.

\section{Introduction}

Litvin (2008) published a six-page paper describing his tables of crystallographic properties of magnetic space groups contained in 4472 pages of supplementary material. The tables describe the 7,80 and 1651 types of Shubnikov space groups in one, two and three dimensions, respectively. Let $\mathbf{G}$ be an ordinary space group, $\mathbf{H}$ a subgroup of index 2 of $\mathbf{G}$ and let $1^{\prime}$ denote time inversion. Among the 1651 types there are 230 of the form $\mathbf{G}, 230$ of the form $\mathbf{G}+\mathbf{G} 1^{\prime}$ and 1191 of the form $\mathbf{H}+$ (G- H)1'. For 674 among the 1191 types $\mathbf{H}$ is an isotranslational subgroup and for the remaining $517 \mathbf{H}$ is an isoclass subgroup. In the language of two-colour groups, where $1^{\prime}$ is interpreted as exchanging black and white (or black and red for typographical reasons), we speak of 230 monochrome types, 230 grey types, 674 black-white types of the first kind and 517 black-white types of the second kind.

Considering a fixed space group G, Litvin (2008), following Opechowski (1986), defines the superfamily of $\mathbf{G}$ consisting of the types containing $\mathbf{G}, \mathbf{G}+\mathbf{G} 1^{\prime}$ and $\mathbf{H}+(\mathbf{G}-\mathbf{H}) 1^{\prime}$ for all subgroups $\mathbf{H}$ of index 2 in G. Koptsik (1966), following Belov et al. $(1957 a, b)$, proceeded differently: They grouped together the types containing $\mathbf{G}, \mathbf{G}+\mathbf{G} 1^{\prime}, \mathbf{H}+(\mathbf{G}-\mathbf{H}) 1^{\prime}$ for all isotranslational subgroups $\mathbf{H}$ of index 2 in $\mathbf{G}$ and $\mathbf{G}+\mathbf{G} \mathbf{t} 1^{\prime}$, where $\mathbf{t}$ is a translation not contained in $\mathbf{G}$ but such that $2 \mathbf{t}$ lies in G. The two definitions group the 1651 types in different ways into 230 'superfamilies'. Both classifications have been used to define symbols for the space-group types that let one immediately recognize the superfamily to which the type belongs. They will be referred to as Opechowski-Guccione (OG) and Belov-Neronova-Smirnova (BNS) symbols. The two symbols agree for monochrome, grey and black-white groups of the first kind (BW1) but differ for black-white groups of the second kind (BW2).
Of prime interest in Litvin (2008) are his descriptions of the 1191 black-white space-group types in three dimensions. Whereas the crystal lattice is determined by all translations contained in $\mathbf{G}$, the point group is isomorphic to the factor group $\mathbf{G} / \mathbf{T}$, where $\mathbf{T}$ is the group of colour-preserving translations in G. The 517 BW2 types have a black-white lattice and a grey point group; they describe antiferromagnets. The 674 BW1 types have a monochrome lattice and a black-white point group; 231 of them admit ferromagnetism, the remaining 443 describe antiferromagnets (see e.g. Borovik-Romanov \& Grimmer, 2003).

Until now the standard work on the 1191 black-white space-group types has been the book by Koptsik (1966), giving for each of these types two diagrams showing a projection of its symmetry elements and of an object in a general position, respectively. Whereas in the diagrams of Koptsik it is often difficult to distinguish between mirror planes and the different kinds of glide planes, Litvin's analogous diagrams are of high graphical quality. Except for the subgroups and supergroups, most other information that Volume A of International Tables for Crystallography (1995) gives for the 230 monochrome types is given by Litvin (2008) in a similar presentation for all 1651 types. Last but not least, most readers will appreciate that Litvin (2008) presents his information in English, whereas Koptsik (1966) used Russian.

The book of Koptsik (1966) being out of print for many years, the gigantic tables of Litvin (2008) are most welcome. In the view of the present author, their main drawback is the use of OG notation. In fact, we shall show that OG notation has decisive disadvantages, which led to the more widespread use of BNS notation. The main aim of the present paper is facilitating the use of Litvin's tables to those familiar with BNS notation.

As an example, let us compare superfamily 67 in the sense of BNS and in the sense of OG. As stated above, the two 


\section{research papers}

Table 1

The Shubnikov space-group types related to point group 222.

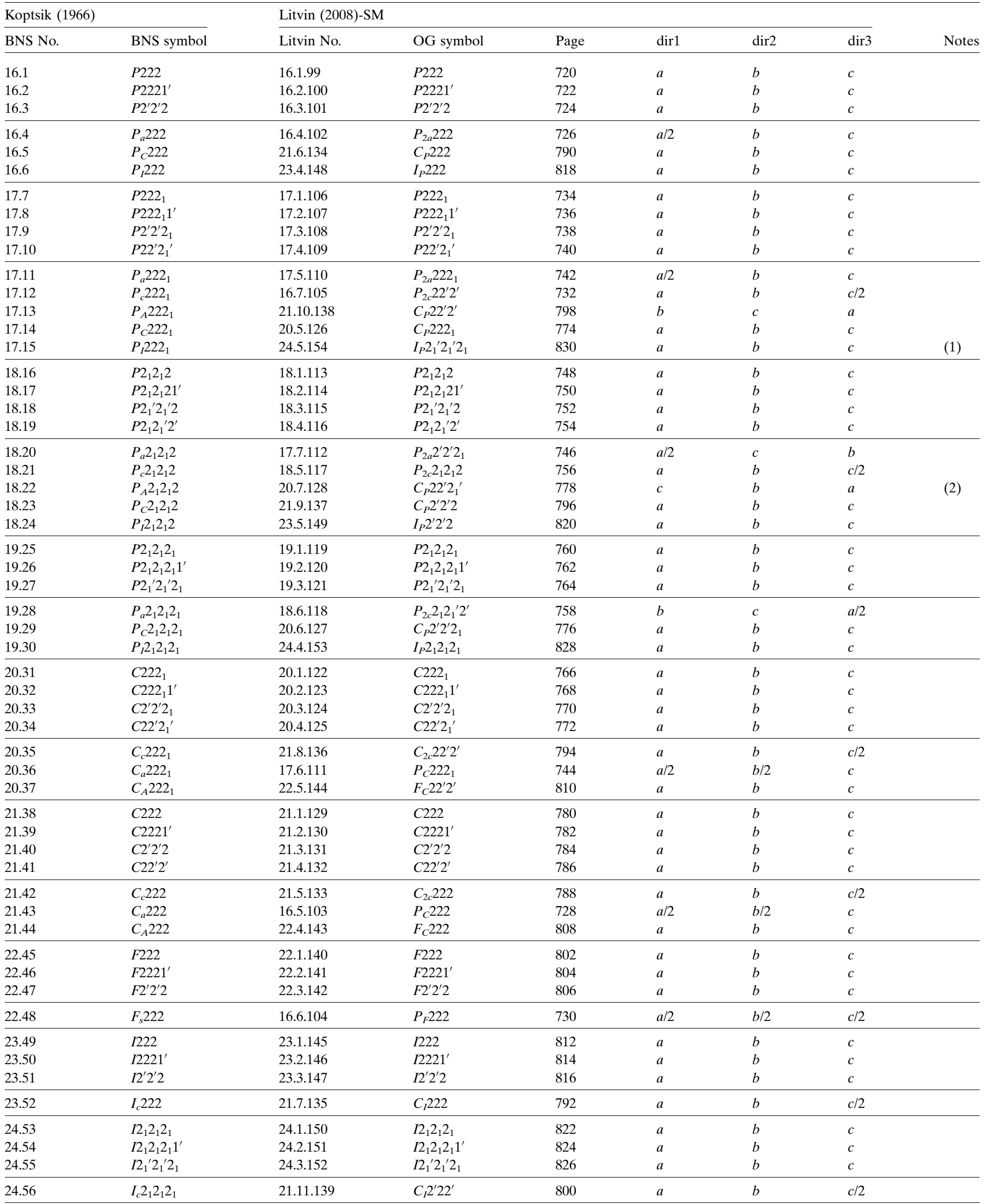

Notes. (1) Litvin (2008)-SM, dir1: black and red exchanged; (2) Koptsik (1966): diagram of symmetry elements wrong. 
Table 2

The BW2 space-group types related to point group $m m 2$.

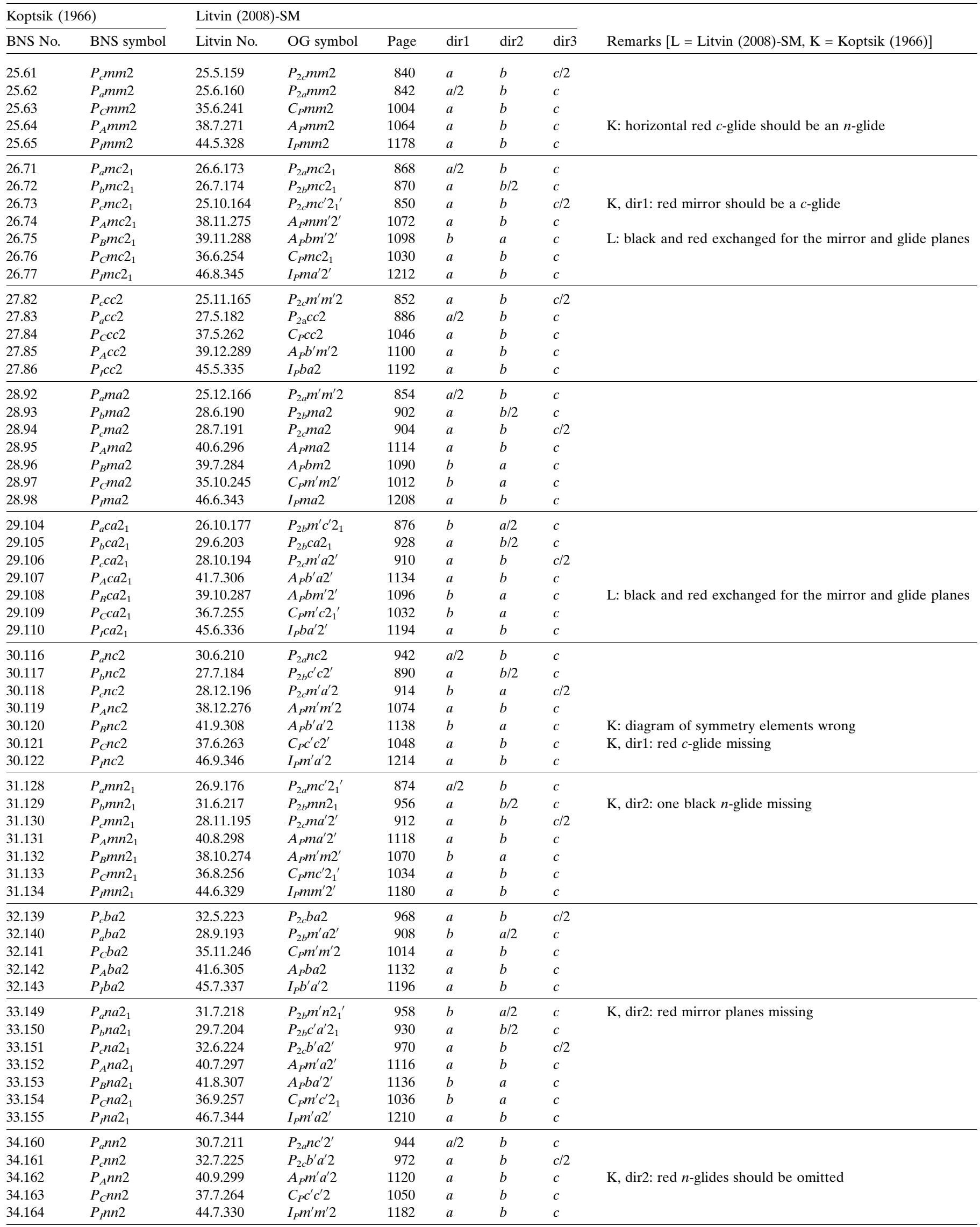


Table 2 (continued)

\begin{tabular}{|c|c|c|c|c|c|c|c|c|}
\hline \multicolumn{2}{|c|}{ Koptsik (1966) } & \multicolumn{6}{|c|}{ Litvin (2008)-SM } & \multirow[b]{2}{*}{ Remarks [L = Litvin (2008)-SM, K = Koptsik (1966)] } \\
\hline BNS No. & BNS symbol & Litvin No. & OG symbol & Page & $\operatorname{dir} 1$ & $\operatorname{dir} 2$ & $\operatorname{dir} 3$ & \\
\hline 35.169 & $C_{c} m m 2$ & 35.5 .240 & $C_{2 c} m m 2$ & 1002 & $a$ & $b$ & $c / 2$ & \\
\hline 35.170 & $C_{a} m m 2$ & 25.7 .161 & $P_{C} m m 2$ & 844 & $a / 2$ & $b / 2$ & $c$ & \\
\hline 35.171 & $C_{A} m m 2$ & 42.5 .313 & $F_{C} m m 2$ & 1148 & $a$ & $b$ & $c$ & \\
\hline 36.177 & $C_{c} m c 2_{1}$ & 35.8 .243 & $C_{2 c} m^{\prime} m 2^{\prime}$ & 1008 & $b$ & $a$ & $c / 2$ & \\
\hline 36.178 & $C_{a} m c 2_{1}$ & 26.8.175 & $P_{C} m c 2_{1}$ & 872 & $a / 2$ & $b / 2$ & $c$ & \\
\hline 36.179 & $C_{A} m c 2_{1}$ & 42.7 .315 & $F_{C} m m^{\prime} 2^{\prime}$ & 1152 & $a$ & $b$ & $c$ & \\
\hline 37.184 & $C_{c} c c 2$ & 35.9 .244 & $C_{2 c} m^{\prime} m^{\prime} 2$ & 1010 & $a$ & $b$ & $c / 2$ & \\
\hline 37.185 & $C_{a} c c 2$ & 27.6 .183 & $P_{C} c c 2$ & 888 & $a / 2$ & $b / 2$ & $c$ & \\
\hline 37.186 & $C_{A} c c 2$ & 42.8 .316 & $F_{C} m^{\prime} m^{\prime} 2$ & 1154 & $a$ & $b$ & $c$ & \\
\hline 38.192 & $A_{a} m m 2$ & 38.6 .270 & $A_{2 a} m m 2$ & 1062 & $a / 2$ & $b$ & $c$ & $\mathrm{~K}$, dir1: red mirror planes missing; L, dir1: omit $n$-glides \\
\hline 38.193 & $A_{c} m m 2$ & 25.8 .162 & $P_{A} m m 2$ & 846 & $a$ & $b / 2$ & $c / 2$ & $\begin{array}{l}\text { dir1: replace } c \text {-glides by } e \text {-glides; } \mathrm{K} \text {, dir2: red mirror planes } \\
\text { missing }\end{array}$ \\
\hline 38.194 & $A_{C} m m 2$ & 42.6 .314 & $F_{A} m m 2$ & 1150 & $a$ & $b$ & $c$ & $\begin{array}{l}\mathrm{K} \text {, dir1: red } e \text {-glides missing; L, dir1: omit } n \text {-glides, replace } \\
\quad \text { axial glides by } e \text {-glides }\end{array}$ \\
\hline 39.200 & $A_{a} b m 2$ & 39.6 .283 & $A_{2 a} b m 2$ & 1088 & $a / 2$ & $b$ & $c$ & dir1: replace axial glides by $e$-glides \\
\hline 39.201 & $A_{c} b m 2$ & 25.13 .167 & $P_{A} m^{\prime} m^{\prime} 2$ & 856 & $a$ & $b / 2$ & $c / 2$ & $\begin{array}{l}\text { dir1: replace axial glides by } e \text {-glides; } \mathrm{K} \text {, dir1: replace } n \text {-glides } \\
\text { by mirrors }\end{array}$ \\
\hline 39.202 & $A_{C} b m 2$ & 42.9 .317 & $F_{A} m^{\prime} m 2^{\prime}$ & 1156 & $a$ & $b$ & $c$ & dir1: replace axial glides by $e$-glides; $\mathrm{L}, \operatorname{dir} 1:$ omit $n$-glides \\
\hline 40.208 & $A_{a} m a 2$ & 38.9 .273 & $A_{2 a} m m^{\prime} 2^{\prime}$ & 1068 & $a / 2$ & $b$ & $c$ & L, dir1: omit $n$-glides \\
\hline 40.209 & $A_{c} m a 2$ & 28.8 .192 & $P_{A} m a 2$ & 906 & $a$ & $b / 2$ & $c / 2$ & dir1: replace axial glides by $e$-glides \\
\hline 40.210 & $A_{C} m a 2$ & 42.10 .318 & $F_{A} m m^{\prime} 2^{\prime}$ & 1158 & $a$ & $b$ & $c$ & dir1: replace axial glides by $e$-glides; L, dir1: omit $n$-glides \\
\hline 41.216 & $A_{a} b a 2$ & 39.9 .286 & $A_{2 a} b^{\prime} m^{\prime} 2$ & 1094 & $a / 2$ & $b$ & $c$ & $\begin{array}{l}\text { dir1: replace axial glides by } e \text {-glides; } \mathrm{L}, \text { dir3: rotations and } \\
\text { screws in top and bottom rows should be black, in middle } \\
\text { row red }\end{array}$ \\
\hline 41.217 & $A_{c} b a 2$ & 28.13.197 & $P_{A} m^{\prime} a^{\prime} 2$ & 916 & $a$ & $b / 2$ & $c / 2$ & dir1: replace axial glides by $e$-glides \\
\hline 41.218 & $A_{C} b a 2$ & 42.11 .319 & $F_{A} m^{\prime} m^{\prime} 2$ & 1160 & $a$ & $b$ & $c$ & dir1: replace axial glides by $e$-glides; L, dir1: omit $n$-glides \\
\hline 42.223 & $F_{s} m m 2$ & 25.9 .163 & $P_{F} m m 2$ & 848 & $a / 2$ & $b / 2$ & $c / 2$ & replace glide planes by $e$-glides \\
\hline 43.228 & $F_{s} d d 2$ & 34.5 .235 & $P_{F} n n 2$ & 992 & $a / 2$ & $b / 2$ & $c / 2$ & \\
\hline 44.233 & $I_{c} m m 2$ & 35.7 .242 & $C_{I} m m 2$ & 1006 & $a$ & $b$ & $c / 2$ & \\
\hline 44.234 & $I_{a} m m 2$ & 38.8 .272 & $A_{I} m m 2$ & 1066 & $a / 2$ & $b$ & $c$ & \\
\hline 45.239 & $I_{c} b a 2$ & 35.13 .248 & $C_{I} m^{\prime} m^{\prime} 2$ & 1018 & $a$ & $b$ & $c / 2$ & \\
\hline 45.240 & $I_{a} b a 2$ & 39.13 .290 & $A_{I} b^{\prime} m^{\prime} 2$ & 1102 & $a / 2$ & $b$ & $c$ & \\
\hline 46.246 & $I_{c} m a 2$ & 35.12 .247 & $C_{I} m^{\prime} m 2^{\prime}$ & 1016 & $a$ & $b$ & $c / 2$ & \\
\hline 46.247 & $I_{a} m a 2$ & 38.13 .277 & $A_{I} m^{\prime} m^{\prime} 2$ & 1076 & $a / 2$ & $b$ & $c$ & $\begin{array}{l}\mathrm{L} \text {, dir2: red } c \text {-glide should be black, black } n \text {-glide should be } \\
\text { red }\end{array}$ \\
\hline 46.248 & $I_{b} m a 2$ & 39.8 .285 & $A_{I} b m 2$ & 1092 & $b / 2$ & $a$ & $c$ & \\
\hline
\end{tabular}

superfamilies contain the same monochrome type (Cmma), the same grey type $\left(C m m a 1^{\prime}\right)$ and the same black-white types of the first kind $\left(\mathrm{Cm}^{\prime} m a, C m m a^{\prime}, C m^{\prime} m^{\prime} a, C m m^{\prime} a^{\prime}, C m^{\prime} m^{\prime} a^{\prime}\right)$.

Superfamily 67 in the sense of BNS contains in addition the following BW2 types: $C_{c} m m a, C_{a} m m a$ and $C_{A} m m a$. Their monochrome subgroups are of type Cmma. Starting with the diagram of symmetry elements for Cmma, the ones for $C_{c} m m a, C_{a} m m a$ and $C_{A} m m a$ are easily obtained by combining each symmetry element in the diagram for Cmma with a subsequent colour-changing translation by $c / 2, a / 2$ and $b / 2+$ $c / 2$, respectively. Similarly, the general-position diagrams for $C_{c} m m a, C_{a} m m a$ and $C_{A} m m a$ are obtained by applying to each position in the diagram for Cmma a colour-changing translation by $c / 2, a / 2$ and $b / 2+c / 2$, respectively. In the general case, there are 16 positions equivalent under Cmma. For 8 of them the coordinate triplets are given explicitly in International Tables as a $(0,0,0)+$ set, the other 8 being obtained by adding $\left(\frac{1}{2}, \frac{1}{2}, 0\right)$. For the interpretation of the BNS symbol it does not matter how the 16 positions are split into a $(0,0,0)+$ and a $\left(\frac{1}{2}, \frac{1}{2}, 0\right)+$ set because the objects in all 16 positions have the same colour.

Superfamily 67 in the sense of OG contains the following BW2 types: $C_{2 c} m m a, C_{2 c} m^{\prime} m a, C_{2 c} m^{\prime} m^{\prime} a, C_{P} m m a, C_{P} m^{\prime} m a$, $C_{P} m m^{\prime} a, C_{P} m m a^{\prime}, C_{I} m m a, C_{I} m m^{\prime} a$ and $C_{I} m^{\prime} m a^{\prime}$. Whereas different BW2 types in a BNS superfamily have different centring symbols and never contain primes, different BW2 types in an OG superfamily may have the same centring symbol but a different arrangement of primes. The meaning of the centring symbols differs in BNS and $\mathrm{OG}$ notation: Whereas in BNS notation the $C$-centred orthorhombic cell is spanned by unprimed translations $a / 2+b / 2, b$ and $c$, in $\mathrm{OG}$ notation it is spanned by $a / 2+b / 2, b$ and $c^{\prime}$ for $C_{2 c}$, by $a^{\prime} / 2+$ $b^{\prime} / 2, b$ and $c$ for $C_{P}$, and by $a^{\prime} / 2+b^{\prime} / 2, b$ and $c^{\prime}$ for $C_{I}$. The elements in the $\mathrm{OG}$ symbol to the right of the centring subscript denote operations mapping position $x, y, z$ into a position in the $(0,0,0)+$ set. The objects at these two positions have the same colour if the element is unprimed and different colours if the element is primed. Now it matters how the 
research papers

Table 3

The monochrome, grey and BW1 space-group types related to point group $m m 2$.

\begin{tabular}{|c|c|c|c|c|}
\hline BNS No. & Symbol & Litvin No. & Page & Notes \\
\hline 25.57 & Pmm2 & 25.1 .155 & 832 & \\
\hline 25.58 & Pmm21' & 25.2 .156 & 834 & \\
\hline 25.59 & $P m^{\prime} m 2^{\prime}$ & 25.3 .157 & 836 & \\
\hline 25.60 & $P m^{\prime} m^{\prime} 2$ & 25.4 .158 & 838 & \\
\hline 26.66 & $P m c 2_{1}$ & 26.1.168 & 858 & \\
\hline 26.67 & $P m c 2_{1} 1^{\prime}$ & 26.2.169 & 860 & \\
\hline 26.68 & $P m^{\prime} c 2_{1}^{\prime}$ & 26.3 .170 & 862 & \\
\hline 26.69 & $P m c^{\prime} 2_{1}^{\prime}$ & 26.4 .171 & 864 & \\
\hline 26.70 & $P m^{\prime} c^{\prime} 2_{1}$ & 26.5 .172 & 866 & \\
\hline 27.78 & $P c c 2$ & 27.1.178 & 878 & \\
\hline 27.79 & Pcc $21^{\prime}$ & 27.2.179 & 880 & \\
\hline 27.80 & $P c^{\prime} c 2^{\prime}$ & 27.3.180 & 882 & \\
\hline 27.81 & $P c^{\prime} c^{\prime} 2$ & 27.4 .181 & 884 & \\
\hline 28.87 & Pma2 & 28.1 .185 & 892 & \\
\hline 28.88 & Pma21' & 28.2 .186 & 894 & \\
\hline 28.89 & $P m^{\prime} a 2^{\prime}$ & 28.3.187 & 896 & \\
\hline 28.90 & $P m a^{\prime} 2^{\prime}$ & 28.4 .188 & 898 & \\
\hline 28.91 & $P m^{\prime} a^{\prime} 2$ & 28.5.189 & 900 & \\
\hline 29.99 & $\mathrm{Pca}_{1}$ & 29.1.198 & 918 & \\
\hline 29.100 & $P c a 2_{1} 1^{\prime}$ & 29.2.199 & 920 & \\
\hline 29.101 & $P c^{\prime} a 2_{1}^{\prime}$ & 29.3.200 & 922 & (1) \\
\hline 29.102 & $P c a^{\prime} 2_{1}^{\prime}$ & 29.4.201 & 924 & (2) \\
\hline 29.103 & $P c^{\prime} a^{\prime} 2_{1}$ & 29.5.202 & 926 & \\
\hline 30.111 & Pnc2 & 30.1 .205 & 932 & \\
\hline 30.112 & Pnc $21^{\prime}$ & 30.2 .206 & 934 & \\
\hline 30.113 & $P n^{\prime} c 2^{\prime}$ & 30.3 .207 & 936 & \\
\hline 30.114 & $P n c^{\prime} 2^{\prime}$ & 30.4 .208 & 938 & \\
\hline 30.115 & $P n^{\prime} c^{\prime} 2$ & 30.5.209 & 940 & \\
\hline 31.123 & $P m n 2_{1}$ & 31.1.212 & 946 & \\
\hline 31.124 & $\operatorname{Pmn} 2_{1} 1^{\prime}$ & 31.2 .213 & 948 & \\
\hline 31.125 & $P m^{\prime} n 2_{1}^{\prime}$ & 31.3.214 & 950 & \\
\hline 31.126 & $P m n^{\prime} 2_{1}^{\prime}$ & 31.4 .215 & 952 & \\
\hline 31.127 & $P m^{\prime} n^{\prime} 2_{1}$ & 31.5 .216 & 954 & \\
\hline 32.135 & $\mathrm{Pba} 2$ & 32.1 .219 & 960 & \\
\hline 32.136 & $P b a 21^{\prime}$ & 32.2 .220 & 962 & \\
\hline 32.137 & $P b^{\prime} a 2^{\prime}$ & 32.3 .221 & 964 & \\
\hline 32.138 & $P b^{\prime} a^{\prime} 2$ & 32.4 .222 & 966 & \\
\hline 33.144 & $\operatorname{Pna}_{1}$ & 33.1 .226 & 974 & \\
\hline 33.145 & $P n a 2_{1} 1^{\prime}$ & 33.2 .227 & 976 & \\
\hline 33.146 & $P n^{\prime} a 2_{1}^{\prime}$ & 33.3 .228 & 978 & \\
\hline 33.147 & $P n a^{\prime} 2_{1}^{\prime}$ & 33.4 .229 & 980 & \\
\hline 33.148 & $P n^{\prime} a^{\prime} 2_{1}$ & 33.5 .230 & 982 & \\
\hline 34.156 & Pnn2 & 34.1 .231 & 984 & \\
\hline 34.157 & Pnn21' & 34.2 .232 & 986 & \\
\hline 34.158 & $P n^{\prime} n 2^{\prime}$ & 34.3 .233 & 988 & \\
\hline 34.159 & $P n^{\prime} n^{\prime} 2$ & 34.4 .234 & 990 & \\
\hline 35.165 & $\mathrm{Cmm} 2$ & 35.1 .236 & 994 & \\
\hline 35.166 & Cmm21' & 35.2 .237 & 996 & \\
\hline 35.167 & $C m^{\prime} m 2^{\prime}$ & 35.3 .238 & 998 & \\
\hline 35.168 & $C m^{\prime} m^{\prime} 2$ & 35.4 .239 & 1000 & \\
\hline 36.172 & $C m c 2_{1}$ & 36.1 .249 & 1020 & \\
\hline 36.173 & $C m c 2{ }_{1} 1^{\prime}$ & 36.2 .250 & 1022 & \\
\hline 36.174 & $C m^{\prime} c 2_{1}^{\prime}$ & 36.3 .251 & 1024 & \\
\hline 36.175 & $C m c^{\prime} 2_{1}^{\prime}$ & 36.4 .252 & 1026 & \\
\hline 36.176 & $C m^{\prime} c^{\prime} 2_{1}$ & 36.5 .253 & 1028 & \\
\hline 37.180 & Ccc2 & 37.1 .258 & 1038 & \\
\hline 37.181 & Ccc $21^{\prime}$ & 37.2 .259 & 1040 & \\
\hline 37.182 & $C c^{\prime} c 2^{\prime}$ & 37.3 .260 & 1042 & (3) \\
\hline 37.183 & $C c^{\prime} c^{\prime} 2$ & 37.4 .261 & 1044 & \\
\hline 38.187 & Amm2 & 38.1 .265 & 1052 & \\
\hline 38.188 & Amm21' & 38.2 .266 & 1054 & \\
\hline 38.189 & $A m^{\prime} m 2^{\prime}$ & 38.3 .267 & 1056 & (4) \\
\hline
\end{tabular}

Table 3 (continued)

\begin{tabular}{|c|c|c|c|c|}
\hline BNS No. & Symbol & Litvin No. & Page & Notes \\
\hline 38.190 & $A m m^{\prime} 2^{\prime}$ & 38.4 .268 & 1058 & $(5)$ \\
\hline 38.191 & $A m^{\prime} m^{\prime} 2$ & 38.5 .269 & 1060 & (4) \\
\hline 39.195 & $A b m 2$ & 39.1 .278 & 1078 & (6) \\
\hline 39.196 & $A b m 21^{\prime}$ & 39.2.279 & 1080 & (6) \\
\hline 39.197 & $A b^{\prime} m 2^{\prime}$ & 39.3 .280 & 1082 & (7) \\
\hline 39.198 & $A b m^{\prime} 2^{\prime}$ & 39.4 .281 & 1084 & (6) \\
\hline 39.199 & $A b^{\prime} m^{\prime} 2$ & 39.5 .282 & 1086 & (7) \\
\hline 40.203 & Ama2 & 40.1 .291 & 1104 & $(5)$ \\
\hline 40.204 & Ama21' & 40.2 .292 & 1106 & (5) \\
\hline 40.205 & $A m^{\prime} a 2^{\prime}$ & 40.3 .293 & 1108 & (4) \\
\hline 40.206 & $A m a^{\prime} 2^{\prime}$ & 40.4 .294 & 1110 & (5) \\
\hline 40.207 & $A m^{\prime} a^{\prime} 2$ & 40.5 .295 & 1112 & (4) \\
\hline 41.211 & $A b a 2$ & 41.1 .300 & 1122 & (6) \\
\hline 41.212 & $A b a 21^{\prime}$ & 41.2 .301 & 1124 & (6) \\
\hline 41.213 & $A b^{\prime} a 2^{\prime}$ & 41.3 .302 & 1126 & (7) \\
\hline 41.214 & $A b a^{\prime} 2^{\prime}$ & 41.4.303 & 1128 & (6) \\
\hline 41.215 & $A b^{\prime} a^{\prime} 2$ & 41.5.304 & 1130 & $(7)$ \\
\hline 42.219 & $F m m 2$ & 42.1 .309 & 1140 & $(8),(9)$ \\
\hline 42.220 & Fmm $21^{\prime}$ & 42.2 .310 & 1142 & $(8),(9)$ \\
\hline 42.221 & $F m^{\prime} m 2^{\prime}$ & 42.3 .311 & 1144 & $(8),(9)$ \\
\hline 42.222 & $F m^{\prime} m^{\prime} 2$ & 42.4 .312 & 1146 & $(8),(9)$ \\
\hline 43.224 & $F d d 2$ & 43.1 .320 & 1162 & \\
\hline 43.225 & $F d d 21^{\prime}$ & 43.2 .321 & 1164 & \\
\hline 43.226 & $F d^{\prime} d 2^{\prime}$ & 43.3 .322 & 1166 & \\
\hline 43.227 & $F d^{\prime} d^{\prime} 2$ & 43.4 .323 & 1168 & \\
\hline 44.229 & $\operatorname{Imm} 2$ & 44.1 .324 & 1170 & \\
\hline 44.230 & $\operatorname{Imm} 21^{\prime}$ & 44.2 .325 & 1172 & \\
\hline 44.231 & $\operatorname{Im} m 2^{\prime}$ & 44.3 .326 & 1174 & \\
\hline 44.232 & $I m^{\prime} m^{\prime} 2$ & 44.4 .327 & 1176 & \\
\hline 45.235 & $I b a 2$ & 45.1 .331 & 1184 & \\
\hline 45.236 & $I b a 21^{\prime}$ & 45.2 .332 & 1186 & \\
\hline 45.237 & $I b^{\prime} a 2^{\prime}$ & 45.3 .333 & 1188 & $(10)$ \\
\hline 45.238 & $I b^{\prime} a^{\prime} 2$ & 45.4 .334 & 1190 & \\
\hline 46.241 & $\operatorname{Ima} 2$ & 46.1 .338 & 1198 & \\
\hline 46.242 & $\operatorname{Ima} 21^{\prime}$ & 46.2 .339 & 1200 & \\
\hline 46.243 & $\operatorname{Im}^{\prime} a 2^{\prime}$ & 46.3 .340 & 1202 & \\
\hline 46.244 & $\operatorname{Im} a^{\prime} 2^{\prime}$ & 46.4.341 & 1204 & \\
\hline 46.245 & $\operatorname{Im}^{\prime} a^{\prime} 2$ & 46.5 .342 & 1206 & \\
\hline
\end{tabular}

Notes. (1) Koptsik (1966): diagrams of symmetry elements unclear; (2) Koptsik (1966): diagrams of symmetry elements misleading; (3) Litvin (2008)-SM: screws should be rotations; (4) Litvin (2008)-SM, dir1: omit red $n$-glide; (5) Litvin (2008)-SM, dir1: omit black $n$-glide; (6) dir1: replace axial glides by black $e$-glide; (7) dir1: replace axial glides by red $e$-glide; (8) Litvin (2008)-SM: omit all $n$-glides; (9) replace axial glides by $e$-glide of the same colour; (10) Litvin (2008)-SM shows $I b a^{\prime} 2^{\prime}$ not $I b^{\prime} a 2^{\prime}$.

triplets for the general position are split into various sets when the conventional lattice is centred. Originally, the meaning of the OG symbols was based on the choice made in Volume I of International Tables for X-ray Crystallography (1952), which will be referred to as ITXC52. Taking instead the choice made in Volume A of International Tables for Crystallography (1983) (which will be referred to as ITC83) as proposed by Litvin (1998), primed and unprimed have to be interchanged in superfamily 67 for $a$ and the second $m$ in the cases of $C_{P}$ and $C_{I}$ according to Table 2 in Litvin (1998). Because this means that one has to specify the edition of International Tables underlying the symbol, Litvin (2001) proposed returning to the original definition of the OG symbols. Whereas ITXC52 or the 216-page supplementary material to Litvin (2001) has to be consulted for interpreting the OG symbols of BW2 types, 
Table 4

The BW2 space-group types related to point group $\mathrm{mmm}$.

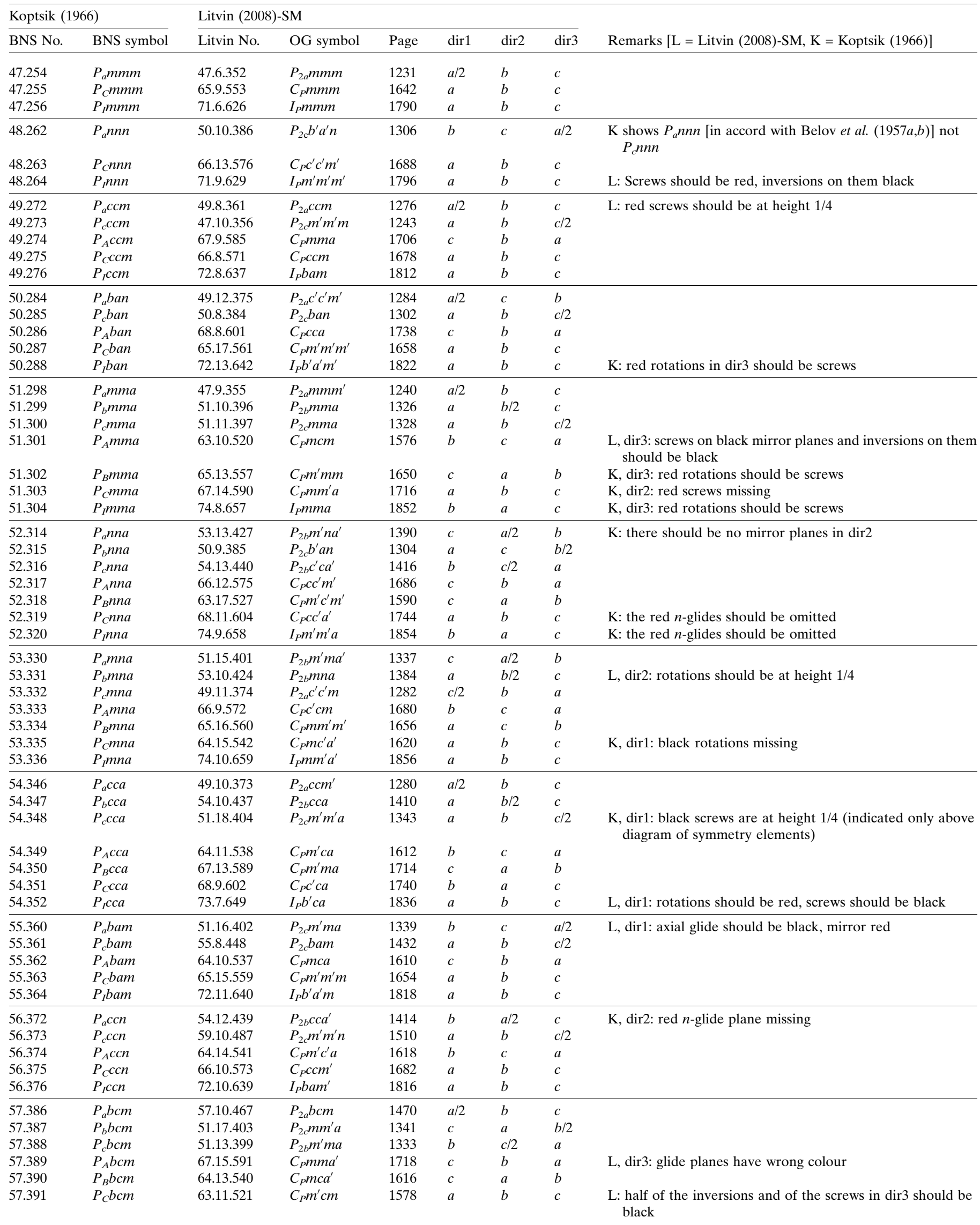


research papers

Table 4 (continued)

\begin{tabular}{|c|c|c|c|c|c|c|c|c|}
\hline \multicolumn{2}{|c|}{ Koptsik (1966) } & \multicolumn{6}{|c|}{ Litvin (2008)-SM } & \multirow[b]{2}{*}{ Remarks [L = Litvin (2008)-SM, K = Koptsik (1966)] } \\
\hline BNS No. & BNS symbol & Litvin No. & OG symbol & Page & $\operatorname{dir} 1$ & $\operatorname{dir} 2$ & $\operatorname{dir} 3$ & \\
\hline 57.392 & $P_{I} b c m$ & 72.9 .638 & $I_{P} b^{\prime} a m$ & 1814 & $a$ & $b$ & $c$ & \\
\hline 58.400 & $P_{a} n n m$ & 53.12 .426 & $P_{2 b} m n a^{\prime}$ & 1388 & $c$ & $a / 2$ & $b$ & \\
\hline 58.401 & $P_{c} n n m$ & 55.10 .450 & $P_{2 c} b^{\prime} a^{\prime} m$ & 1436 & $a$ & $b$ & $c / 2$ & L: red (not black) inversion points lie at height $1 / 2$ \\
\hline 58.402 & $P_{A} n n m$ & 63.15 .525 & $C_{P} m c^{\prime} m^{\prime}$ & 1586 & $c$ & $b$ & $a$ & \\
\hline 58.403 & $P_{C} n n m$ & 66.11 .574 & $C_{P} c^{\prime} c^{\prime} m$ & 1684 & $a$ & $b$ & $c$ & \\
\hline 58.404 & $P_{I} n n m$ & 71.8 .628 & $I_{P} m^{\prime} m^{\prime} m$ & 1794 & $a$ & $b$ & $c$ & \\
\hline 59.412 & $P_{a} m m n$ & 51.14 .400 & $P_{2 b} m m a^{\prime}$ & 1335 & $b$ & $a / 2$ & $c$ & \\
\hline 59.413 & $P_{c} m m n$ & 59.8 .485 & $P_{2 c} m m n$ & 1506 & $a$ & $b$ & $c / 2$ & \\
\hline 59.414 & $P_{A} m m n$ & 63.12 .522 & $C_{P} m c^{\prime} m$ & 1580 & $b$ & $c$ & $a$ & \\
\hline 59.415 & $P_{C} m m n$ & 65.14 .558 & $C_{P} m m m^{\prime}$ & 1652 & $a$ & $b$ & $c$ & $\mathrm{~K}$ : inversion on black rotation should be red \\
\hline 59.416 & $P_{I} m m n$ & 71.7 .627 & $I_{P} m^{\prime} m m$ & 1792 & $c$ & $a$ & $b$ & L, dir2: $n$-glides should be red \\
\hline 60.426 & $P_{a} b c n$ & 54.11 .438 & $P_{2 b} c^{\prime} c a$ & 1412 & $c$ & $a / 2$ & $b$ & \\
\hline 60.427 & $P_{b} b c n$ & 57.13 .470 & $P_{2 a} b c^{\prime} m^{\prime}$ & 1476 & $b / 2$ & $c$ & $a$ & \\
\hline 60.428 & $P_{c} b c n$ & 53.11 .425 & $P_{2 b} m^{\prime} n a$ & 1386 & $b$ & $c / 2$ & $a$ & \\
\hline 60.429 & $P_{A} b c n$ & 64.17 .544 & $C_{P} m^{\prime} c^{\prime} a^{\prime}$ & 1624 & $b$ & $c$ & $a$ & $\mathrm{~K}$, dir1: red axial glide planes missing \\
\hline 60.430 & $P_{B} b c n$ & 68.10 .603 & $C_{P} c c a^{\prime}$ & 1742 & $c$ & $a$ & $b$ & \\
\hline 60.431 & $P_{C} b c n$ & 63.16 .526 & $C_{P} m^{\prime} \mathrm{cm}^{\prime}$ & 1588 & $a$ & $b$ & $c$ & \\
\hline 60.432 & $P_{I} b c n$ & 72.12 .641 & $I_{P} b^{\prime} a m^{\prime}$ & 1820 & $a$ & $b$ & $c$ & $\mathrm{~K}$, dir2: red axial glide planes missing \\
\hline 61.438 & $P_{a} b c a$ & 57.12 .469 & $P_{2 a} b c m^{\prime}$ & 1474 & $a / 2$ & $b$ & $c$ & $\mathrm{~K}$, dir2: red $n$-glide planes missing \\
\hline 61.439 & $P_{C} b c a$ & 64.16 .543 & $C_{P} m^{\prime} c a^{\prime}$ & 1622 & $a$ & $b$ & $c$ & \\
\hline 61.440 & $P_{I} b c a$ & 73.6 .648 & $I_{P} b c a$ & 1834 & $a$ & $b$ & $c$ & $\mathrm{~K}$, dir2: red rotations lie at height 0 \\
\hline 62.450 & $P_{a} n m a$ & 59.9 .486 & $P_{2 c} m^{\prime} m n$ & 1508 & $c$ & $b$ & $a / 2$ & \\
\hline 62.451 & $P_{b} n m a$ & 55.9 .449 & $P_{2 c} b^{\prime} a m$ & 1434 & $a$ & $c$ & $b / 2$ & \\
\hline 62.452 & $P_{c} n m a$ & 57.11 .468 & $P_{2 a} b c^{\prime} m$ & 1472 & $c / 2$ & $a$ & $b$ & \\
\hline 62.453 & $P_{A} n m a$ & 63.13 .523 & $C_{P} m c m^{\prime}$ & 1582 & $b$ & $c$ & $a$ & \\
\hline 62.454 & $P_{B} n m a$ & 63.14 .524 & $C_{P} m^{\prime} c^{\prime} m$ & 1584 & $c$ & $a$ & $b$ & \\
\hline 62.455 & $P_{C} n m a$ & 64.12 .539 & $C_{P} m c^{\prime} a$ & 1614 & $b$ & $a$ & $c$ & \\
\hline 62.456 & $P_{I} n m a$ & 74.11 .660 & $I_{P} m^{\prime} m a^{\prime}$ & 1858 & $a$ & $b$ & $c$ & \\
\hline 63.466 & $C_{c} m c m$ & 65.12 .556 & $C_{2 c} m m^{\prime} m^{\prime}$ & 1648 & $a$ & $b$ & $c / 2$ & L \& K: omit $n$-glides in $\operatorname{dir} 3$ \\
\hline 63.467 & $C_{a} m c m$ & 51.12 .398 & $P_{A} m m a$ & 1330 & $c$ & $a / 2$ & $b / 2$ & $\begin{array}{l}\text { L, dir1: no black } n \text {-glide, red glides should be } e \text {-glide; dir } 2 \text {, } \\
\text { height } 1 / 2 \text { : black screw and red rotation missing; dir3: black } \\
n \text {-glide at height } 1 / 2 \text { and red } n \text {-glide missing; K: omit black } \\
n \text {-glide in dir } 3\end{array}$ \\
\hline 63.468 & $C_{A} m c m$ & 69.7 .611 & $F_{C} m^{\prime} m m$ & 1759 & $b$ & $a$ & $c$ & L \& K: omit black $n$-glide in $\operatorname{dir} 3$ \\
\hline 64.478 & $C_{c} m c a$ & 67.11 .587 & $C_{2 c} m^{\prime} m a$ & 1710 & $b$ & $a$ & $c / 2$ & \\
\hline 64.479 & $C_{a} m c a$ & 51.19 .405 & $P_{A} m^{\prime} m a$ & 1345 & $c$ & $a / 2$ & $b / 2$ & $\begin{array}{l}\text { K: some red inversion points marked incorrectly, omit red } \\
n \text {-glide in dir } 3 \text {; L: dir1 black glide should be } e \text {-glide, omit } \\
\text { red } n \text {-glide; dir } 2 \text {, height } 1 / 2 \text { : black rotation and red screw } \\
\text { missing; dir } 3 \text { : red } n \text {-glide at height } 0 \text { and black } n \text {-glide at } \\
\text { height } 1 / 2 \text { missing }\end{array}$ \\
\hline 64.480 & $C_{A} m c a$ & 69.10 .614 & $F_{C} m m^{\prime} m^{\prime}$ & 1765 & $a$ & $b$ & $c$ & L: omit red $n$-glide in dir 3 \\
\hline 65.488 & $C_{c} m m m$ & 65.8 .552 & $C_{2 c} m m m$ & 1640 & $a$ & $b$ & $c / 2$ & L \& K: omit $n$-glides in $\operatorname{dir} 3$ \\
\hline 65.489 & $C_{a} m m m$ & 47.7 .353 & $P_{C} m m m$ & 1234 & $a / 2$ & $b / 2$ & $c$ & $\mathrm{~K}$ : omit black $n$-glide in dir 3 \\
\hline 65.490 & $C_{A} m m m$ & 69.6 .610 & $F_{C} m m m$ & 1757 & $a$ & $b$ & $c$ & $\begin{array}{l}\text { L \& K: omit } n \text {-glide in dir3; K: replace red glides in dir3 by an } \\
e \text {-glide }\end{array}$ \\
\hline 66.498 & $C_{c} c c m$ & 65.11 .555 & $C_{2 c} m^{\prime} m^{\prime} m$ & 1646 & $a$ & $b$ & $c / 2$ & L \& K: omit $n$-glides in $\operatorname{dir} 3$ \\
\hline 66.499 & $C_{a} c c m$ & 49.9 .372 & $P_{C} c c m$ & 1278 & $a / 2$ & $b / 2$ & $c$ & $\begin{array}{l}\mathrm{K} \text { : omit black } n \text {-glide in } \operatorname{dir} 3 ; \mathrm{L}, \operatorname{dir} 1, \operatorname{dir} 2 \text { : every other black } \\
\text { axial glide should be an } n \text {-glide, the red glides are missing }\end{array}$ \\
\hline 66.500 & $C_{A} c c m$ & 69.9 .613 & $F_{C} m^{\prime} m^{\prime} m$ & 1763 & $a$ & $b$ & $c$ & L \& K: omit black $n$-glides in $\operatorname{dir} 3$ \\
\hline 67.508 & $C_{c} m m a$ & 67.8 .584 & $C_{2 c} m m a$ & 1704 & $a$ & $b$ & $c / 2$ & $\mathrm{~K}$, dir1, dir2: red mirror and glide planes wrong \\
\hline 67.509 & $C_{a} m m a$ & 47.11 .357 & $P_{C} m m m^{\prime}$ & 1246 & $a / 2$ & $b / 2$ & $c$ & $\begin{array}{l}\text { K: omit red } n \text {-glide in dir3; L: axial glide in dir3 should be an } \\
e \text {-glide }\end{array}$ \\
\hline 67.510 & $C_{A} m m a$ & 69.8 .612 & $F_{C} m m m^{\prime}$ & 1761 & $a$ & $b$ & $c$ & $\begin{array}{l}\text { L \& K: omit red } n \text {-glide in } \operatorname{dir} 3 ; \mathrm{K} \text {, dir } 1 \text {, dir2: red mirror and } \\
\text { glide planes wrong }\end{array}$ \\
\hline 68.518 & $C_{c} c c a$ & 67.12 .588 & $C_{2 c} m^{\prime} m^{\prime} a$ & 1712 & $a$ & $b$ & $c / 2$ & \\
\hline 68.519 & $C_{a} c c a$ & 49.13 .376 & $P_{C} c c^{\prime}$ & 1286 & $a / 2$ & $b / 2$ & $c$ & L \& K: omit red $n$-glide in $\operatorname{dir} 3$ \\
\hline 68.520 & $C_{A} c c a$ & 69.11 .615 & $F_{C} m^{\prime} m^{\prime} m^{\prime}$ & 1767 & $a$ & $b$ & $c$ & L \& K: omit red $n$-glide in $\operatorname{dir} 3$ \\
\hline 69.526 & $F_{s} m m m$ & 47.8 .354 & $P_{F} m m m$ & 1237 & $a / 2$ & $b / 2$ & $c / 2$ & $\begin{array}{l}\text { L: omit } n \text {-glides in dir1, dir2; rotations and screws in dir2 at } \\
\text { height } 1 / 2 \text { have wrong colour; L \& K: replace axial glides by } \\
\text { e-glide in dir1, dir } 2\end{array}$ \\
\hline 70.532 & $F_{s} d d d$ & 48.6 .363 & $P_{F} n n n$ & 1259 & $a / 2$ & $b / 2$ & $c / 2$ & \\
\hline 71.538 & $I_{c} m m m$ & 65.10 .554 & $C_{I} m m m$ & 1644 & $a$ & $b$ & $c / 2$ & \\
\hline 72.546 & $I_{c} b a m$ & 65.19 .563 & $C_{I} m^{\prime} m^{\prime} m$ & 1662 & $a$ & $b$ & $c / 2$ & \\
\hline
\end{tabular}


Table 4 (continued)

\begin{tabular}{|c|c|c|c|c|c|c|c|c|}
\hline \multicolumn{2}{|c|}{ Koptsik (1966) } & \multicolumn{6}{|c|}{ Litvin (2008)-SM } & \multirow[b]{2}{*}{ Remarks [L = Litvin (2008)-SM, K = Koptsik (1966)] } \\
\hline BNS No. & BNS symbol & Litvin No. & OG symbol & Page & dir1 & $\operatorname{dir} 2$ & $\operatorname{dir} 3$ & \\
\hline 72.547 & $I_{a} b a m$ & 67.10 .586 & $C_{I} m m a$ & 1708 & $c$ & $b$ & $a / 2$ & $\begin{array}{l}\text { L: dir2: rotations and screws have wrong colour; dir3: glide } \\
\text { planes at height } 1 / 2 \text { have wrong colour }\end{array}$ \\
\hline 73.553 & $I_{c} b c a$ & 67.17 .593 & $C_{I} m^{\prime} m a^{\prime}$ & 1722 & $a$ & $b$ & $c / 2$ & \\
\hline 74.561 & $I_{c} m m a$ & 67.16 .592 & $C_{I} m m^{\prime} a$ & 1720 & $a$ & $b$ & $c / 2$ & \\
\hline 74.562 & $I_{a} m m a$ & 65.18 .562 & $C_{I} m^{\prime} m m$ & 1660 & $c$ & $b$ & $a / 2$ & \\
\hline
\end{tabular}

the BNS symbols have the great advantage that their centring symbol relates the BW2 types straightforwardly to the corresponding monochrome type. The interpretation being much more involved for the OG symbols than for the BNS symbols of the BW2 types, errors appear more likely: corrections to the original association of primes to OG symbols of BW2 types in Opechowski \& Guccione (1965) were made by Opechowski (1986) and in the supplementary material to Litvin (2008). Moreover, in the supplementary materials to Litvin (2001, 2008) the definition of the OG symbols for the superfamilies 73 and 206 is based on ITC83, not, as intended, on ITXC52.

In conclusion, it seems unfortunate that OG symbols were proposed as an alternative to the earlier-introduced BNS symbols, also because looking at a symbol of a BW2 type, one often does not know whether it is an OG or a BNS symbol. As an example, the BNS symbol $P_{C} m m 2$ corresponds to the OG symbol $C_{P} m m 2$ and the $\mathrm{OG}$ symbol $P_{C} m m 2$ corresponds to the BNS symbol $C_{a} m m 2$.

To see how the supplementary material to Litvin (2008), which we shall refer to as Litvin (2008)-SM, can be used by those familiar with BNS notation, we have studied the diagrams of symmetry elements for orthorhombic Shubnikov space groups in Litvin's tables and compared them to those in Koptsik (1966). In the fourth edition of Volume A of International Tables for Crystallography (1995), which will be referred to as ITC95, a new graphical symbol was introduced for 'double' glide planes $e$, not parallel to the plane of projection. In Tables 2-5 it will be shown in the last column which diagrams in Koptsik (1966) and in Litvin (2008)-SM are affected by these new symbols. The last column of Tables 1-5 also gives corrections to the diagrams of both authors.

\section{Shubnikov space groups related to point group 222}

Table 1 lists the orthorhombic Shubnikov space-group types without mirror or glide planes. The first part of the BNS number gives the number of the BNS superfamily, the second part numerates the 562 orthorhombic Shubnikov space groups; the first part of the Litvin number gives the number of the OG superfamily, the middle part numerates the Shubnikov space groups within an OG superfamily, the last part numerates the 1651 Shubnikov space groups. The column 'Page' indicates the page in Litvin (2008)-SM on which the description of the Shubnikov space group starts. dir1, dir2 and dir3 refer to the directions downwards, to the right and towards the reader in the diagrams of symmetry elements (DSEs) given in
Litvin (2008)-SM. The conventional orthorhombic cell has lattice parameters $a, b$ and $c$, respectively, in the first, second and third symmetry direction of the BNS symbol. An entry $a, b$ or $c$ in 'dir1' states that the downwards extension of the DSE in Litvin (2008)-SM corresponds to the lattice parameter $a, b$ or $c$; an entry $a / 2, b / 2$ or $c / 2$ states that the downwards extension of the DSE in Litvin (2008)-SM corresponds to $a / 2$, $b / 2$ or $c / 2$. The situation is analogous for 'dir2' and 'dir3'. If $c / 2$ appears in 'dir3' then the height indications appearing in Litvin (2008)-SM are twice as big as those in Koptsik (1966); similarly $a / 2$ or $b / 2$ in 'dir3' mean that the height indications in Litvin (2008)-SM are expressed as fractions of $a / 2$ or $b / 2$, not of $a$ or $b$.

The arrangement of Shubnikov space-group types within a BNS superfamily is always the same: First appears the monochrome type (M), then the grey type $(\mathrm{G})$, then the BW1 types, and finally the BW2 types. Horizontal lines separate the BW2 types in Table 1 from the other types. Notice that for the $\mathrm{M}, \mathrm{G}$ and BW1 types the BNS and OG symbols agree and we have $\operatorname{dir} 1=a, \operatorname{dir} 2=b, \operatorname{dir} 3=c$. For this reason, the tables of orthorhombic groups related to $\mathrm{mm} 2$ and $\mathrm{mmm}$ will be split into two: one for the BW2 types and one for the remaining types.

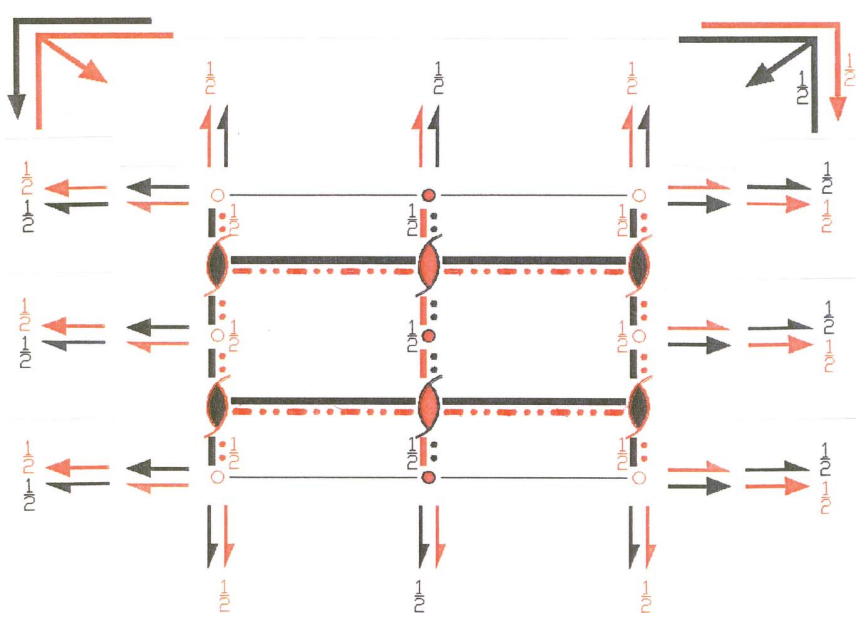

Figure 1

Illustration of the remarks in Table 4 to the diagram of symmetry elements given by Litvin (2008)-SM for type 63.467 (Litvin number 51.12.398, OG symbol $\left.P_{A} m m a\right)$. The symmetry elements in directions 2 and 3 missing in Litvin (2008)-SM have been added; the representation of the symmetry elements in direction 1 has been simplified, thus avoiding the necessity of splitting the diagram into two figures. 
research papers

Table 5

The monochrome, grey and BW1 space-group types related to point group $\mathrm{mmm}$.

\begin{tabular}{|c|c|c|c|c|}
\hline BNS No. & Symbol & Litvin No. & Page & Notes \\
\hline 47.249 & Pmmm & 47.1 .347 & 1216 & \\
\hline 47.250 & Pmmm $1^{\prime}$ & 47.2 .348 & 1219 & \\
\hline 47.251 & $P m^{\prime} m m$ & 47.3 .349 & 1222 & \\
\hline 47.252 & $P m^{\prime} m^{\prime} m$ & 47.4 .350 & 1225 & \\
\hline 47.253 & $P m^{\prime} m^{\prime} m^{\prime}$ & 47.5 .351 & 1228 & \\
\hline 48.257 & Pnnn & 48.1 .358 & 1249 & \\
\hline 48.258 & Pnnn1' & 48.2 .359 & 1251 & \\
\hline 48.259 & $P n^{\prime} n n$ & 48.3 .360 & 1253 & \\
\hline 48.260 & $P n^{\prime} n^{\prime} n$ & 48.4.361 & 1255 & \\
\hline 48.261 & $P n^{\prime} n^{\prime} n^{\prime}$ & 48.5 .362 & 1257 & \\
\hline 49.265 & Pccm & 49.1.364 & 1262 & \\
\hline 49.266 & $P c c m 1^{\prime}$ & 49.2 .365 & 1264 & \\
\hline 49.267 & $P c^{\prime} \mathrm{cm}$ & 49.3.366 & 1266 & (1) \\
\hline 49.268 & Pccm $^{\prime}$ & 49.4.367 & 1268 & $(2)$ \\
\hline 49.269 & $P c^{\prime} c^{\prime} m$ & 49.5 .368 & 1270 & \\
\hline 49.270 & $P c^{\prime} \mathrm{cm}^{\prime}$ & 49.6.369 & 1272 & (3) \\
\hline 49.271 & $P c^{\prime} c^{\prime} m^{\prime}$ & 49.7.360 & 1274 & \\
\hline 50.277 & Pban & 50.1 .377 & 1288 & \\
\hline 50.278 & Pban $1^{\prime}$ & 50.2 .378 & 1290 & \\
\hline 50.279 & $P b^{\prime} a n$ & 50.3 .379 & 1292 & \\
\hline 50.280 & Pban' $^{\prime}$ & 50.4 .380 & 1294 & \\
\hline 50.281 & $P b^{\prime} a^{\prime} n$ & 50.5 .381 & 1296 & \\
\hline 50.282 & $P b^{\prime} a n^{\prime}$ & 50.6 .382 & 1298 & \\
\hline 50.283 & $P b^{\prime} a^{\prime} n^{\prime}$ & 50.7 .383 & 1300 & \\
\hline 51.289 & Pmma & 51.1.387 & 1308 & \\
\hline 51.290 & Pmma1' & 51.2.388 & 1310 & \\
\hline 51.291 & $P m^{\prime} m a$ & 51.3.389 & 1312 & \\
\hline 51.292 & Pmm'a & 51.4 .390 & 1314 & \\
\hline 51.293 & $P m m a^{\prime}$ & 51.5.391 & 1316 & \\
\hline 51.294 & $P m^{\prime} m^{\prime} a$ & 51.6.392 & 1318 & \\
\hline 51.295 & $P m m^{\prime} a^{\prime}$ & 51.7 .393 & 1320 & (4) \\
\hline 51.296 & $P m^{\prime} m a^{\prime}$ & 51.8.394 & 1322 & \\
\hline 51.297 & $P m^{\prime} m^{\prime} a^{\prime}$ & 51.9 .395 & 1324 & \\
\hline 52.305 & Pnna & 52.1 .406 & 1348 & \\
\hline 52.306 & Pnna1' & 52.2 .407 & 1350 & \\
\hline 52.307 & $P n^{\prime} n a$ & 52.3 .408 & 1352 & (5) \\
\hline 52.308 & $P n n^{\prime} a$ & 52.4 .409 & 1354 & $(5)$ \\
\hline 52.309 & Pnna' & 52.5 .410 & 1356 & (5) \\
\hline 52.310 & $P n^{\prime} n^{\prime} a$ & 52.6 .411 & 1358 & (5) \\
\hline 52.311 & $P n n^{\prime} a^{\prime}$ & 52.7 .412 & 1360 & $(5),(6)$ \\
\hline 52.312 & $P n^{\prime} n a^{\prime}$ & 52.8 .413 & 1362 & $(5),(7)$ \\
\hline 52.313 & $P n^{\prime} n^{\prime} a^{\prime}$ & 52.9 .414 & 1364 & (5) \\
\hline 53.321 & Pmna & 53.1 .415 & 1366 & \\
\hline 53.322 & Pmna $1^{\prime}$ & 53.2 .416 & 1368 & \\
\hline 53.323 & $P m^{\prime} n a$ & 53.3 .417 & 1370 & \\
\hline 53.324 & $P m n^{\prime} a$ & 53.4 .418 & 1372 & \\
\hline 53.325 & Pmna' & 53.5.419 & 1374 & \\
\hline 53.326 & $P m^{\prime} n^{\prime} a$ & 53.6 .420 & 1376 & \\
\hline 53.327 & $P m n^{\prime} a^{\prime}$ & 53.7.421 & 1378 & \\
\hline 53.328 & $P m^{\prime} n a^{\prime}$ & 53.8 .422 & 1380 & \\
\hline 53.329 & $P m^{\prime} n^{\prime} a^{\prime}$ & 53.9 .423 & 1382 & \\
\hline 54.337 & Pcca & 54.1 .428 & 1392 & \\
\hline 54.338 & Pcca1 $1^{\prime}$ & 54.2 .429 & 1394 & \\
\hline 54.339 & $P c^{\prime} c a$ & 54.3 .430 & 1396 & \\
\hline 54.340 & $P c c^{\prime} a$ & 54.4 .431 & 1398 & \\
\hline 54.341 & $P c c a^{\prime}$ & 54.5 .432 & 1400 & \\
\hline 54.342 & $P c^{\prime} c^{\prime} a$ & 54.6 .433 & 1402 & \\
\hline 54.343 & $P c c^{\prime} a^{\prime}$ & 54.7 .434 & 1404 & \\
\hline 54.344 & $P c^{\prime} c a^{\prime}$ & 54.8 .435 & 1406 & \\
\hline 54.345 & $P c^{\prime} c^{\prime} a^{\prime}$ & 54.9 .436 & 1408 & \\
\hline 55.353 & Pbam & 55.1 .441 & 1418 & \\
\hline 55.354 & Pbam $1^{\prime}$ & 55.2 .442 & 1420 & \\
\hline
\end{tabular}

Table 5 (continued)

\begin{tabular}{|c|c|c|c|c|}
\hline BNS No. & Symbol & Litvin No. & Page & Notes \\
\hline 55.355 & $P b^{\prime} a m$ & 55.3 .443 & 1422 & \\
\hline 55.356 & Pbam $^{\prime}$ & 55.4 .444 & 1424 & \\
\hline 55.357 & $P b^{\prime} a^{\prime} m$ & 55.5 .445 & 1426 & \\
\hline 55.358 & $P b^{\prime} a m^{\prime}$ & 55.6 .446 & 1428 & \\
\hline 55.359 & $P b^{\prime} a^{\prime} m^{\prime}$ & 55.7 .447 & 1430 & \\
\hline 56.365 & Pccn & 56.1 .451 & 1438 & \\
\hline 56.366 & Pccn $1^{\prime}$ & 56.2 .452 & 1440 & \\
\hline 56.367 & $P c^{\prime} c n$ & 56.3 .453 & 1442 & \\
\hline 56.368 & Pccn' & 56.4 .454 & 1444 & \\
\hline 56.369 & $P c^{\prime} c^{\prime} n$ & 56.5 .455 & 1446 & \\
\hline 56.370 & $P c^{\prime} c n^{\prime}$ & 56.6 .456 & 1448 & \\
\hline 56.371 & $P c^{\prime} c^{\prime} n^{\prime}$ & 56.7 .457 & 1450 & \\
\hline 57.377 & Pbcm & 57.1 .458 & 1452 & \\
\hline 57.378 & $P b c m 1^{\prime}$ & 57.2 .459 & 1454 & \\
\hline 57.379 & $P b^{\prime} \mathrm{cm}$ & 57.3 .460 & 1456 & \\
\hline 57.380 & $P b c^{\prime} m$ & 57.4 .461 & 1458 & \\
\hline 57.381 & $\mathrm{Pbcm}^{\prime}$ & 57.5.462 & 1460 & \\
\hline 57.382 & $P b^{\prime} c^{\prime} m$ & 57.6 .463 & 1462 & \\
\hline 57.383 & $P b c^{\prime} m^{\prime}$ & 57.7 .464 & 1464 & \\
\hline 57.384 & $P b^{\prime} \mathrm{cm}^{\prime}$ & 57.8 .465 & 1466 & \\
\hline 57.385 & $P b^{\prime} c^{\prime} m^{\prime}$ & 57.9.466 & 1468 & $(8)$ \\
\hline 58.393 & Pnnm & 58.1 .471 & 1478 & \\
\hline 58.394 & Pnnm1' & 58.2 .472 & 1480 & \\
\hline 58.395 & $P n^{\prime} n m$ & 58.3 .473 & 1482 & \\
\hline 58.396 & Pnnm' & 58.4 .474 & 1484 & \\
\hline 58.397 & $P n^{\prime} n^{\prime} m$ & 58.5.475 & 1486 & \\
\hline 58.398 & $P n n^{\prime} m^{\prime}$ & 58.6 .476 & 1488 & \\
\hline 58.399 & $P n^{\prime} n^{\prime} m^{\prime}$ & 58.7 .477 & 1490 & \\
\hline 59.405 & Pmmn & 59.1 .478 & 1492 & \\
\hline 59.406 & Pmmn $1^{\prime}$ & 59.2 .479 & 1494 & \\
\hline 59.407 & $P m^{\prime} m n$ & 59.3.480 & 1496 & \\
\hline 59.408 & Pmmn' & 59.4.481 & 1498 & \\
\hline 59.409 & $P m^{\prime} m^{\prime} n$ & 59.5.482 & 1500 & \\
\hline 59.410 & $P m m^{\prime} n^{\prime}$ & 59.6 .483 & 1502 & \\
\hline 59.411 & $P m^{\prime} m^{\prime} n^{\prime}$ & 59.7 .484 & 1504 & \\
\hline 60.417 & Pbcn & 60.1 .488 & 1512 & \\
\hline 60.418 & Pbcn $1^{\prime}$ & 60.2 .489 & 1514 & \\
\hline 60.419 & $P b^{\prime} c n$ & 60.3 .490 & 1516 & \\
\hline 60.420 & $P b c^{\prime} n$ & 60.4 .491 & 1518 & (9) \\
\hline 60.421 & $P b c n^{\prime}$ & 60.5 .492 & 1520 & \\
\hline 60.422 & $P b^{\prime} c^{\prime} n$ & 60.6 .493 & 1522 & \\
\hline 60.423 & $P b c^{\prime} n^{\prime}$ & 60.7 .494 & 1524 & \\
\hline 60.424 & $P b^{\prime} c n^{\prime}$ & 60.8 .495 & 1526 & \\
\hline 60.425 & $P b^{\prime} c^{\prime} n^{\prime}$ & 60.9.496 & 1528 & \\
\hline 61.433 & $P b c a$ & 61.1.497 & 1530 & \\
\hline 61.434 & $P b c a 1^{\prime}$ & 61.2.498 & 1532 & \\
\hline 61.435 & $P b^{\prime} c a$ & 61.3.499 & 1534 & \\
\hline 61.436 & $P b^{\prime} c^{\prime} a$ & 61.4 .500 & 1536 & \\
\hline 61.437 & $P b^{\prime} c^{\prime} a^{\prime}$ & 61.5.501 & 1538 & \\
\hline 62.441 & Pnma & 62.1 .502 & 1540 & \\
\hline 62.442 & Pnma1' & 62.2 .503 & 1542 & \\
\hline 62.443 & $P n^{\prime} m a$ & 62.3 .504 & 1544 & (10) \\
\hline 62.444 & Pnm'a & 62.4 .505 & 1546 & \\
\hline 62.445 & Pnma' & 62.5.506 & 1548 & \\
\hline 62.446 & $P n^{\prime} m^{\prime} a$ & 62.6 .507 & 1550 & \\
\hline 62.447 & $P n m^{\prime} a^{\prime}$ & 62.7 .508 & 1552 & (11) \\
\hline 62.448 & $P n^{\prime} m a^{\prime}$ & 62.8 .509 & 1554 & \\
\hline 62.449 & $P n^{\prime} m^{\prime} a^{\prime}$ & 62.9 .510 & 1556 & \\
\hline 63.457 & $\mathrm{Cmcm}$ & 63.1.511 & 1558 & \\
\hline 63.458 & Cmcm $1^{\prime}$ & 63.2 .512 & 1560 & \\
\hline 63.459 & $\mathrm{Cm}^{\prime} \mathrm{cm}$ & 63.3.513 & 1562 & (12) \\
\hline 63.460 & $C m c^{\prime} m$ & 63.4 .514 & 1564 & (12) \\
\hline 63.461 & $\mathrm{Cmcm}^{\prime}$ & 63.5.515 & 1566 & (12) \\
\hline 63.462 & $C m^{\prime} c^{\prime} m$ & 63.6.516 & 1568 & (12) \\
\hline
\end{tabular}


Table 5 (continued)

\begin{tabular}{|c|c|c|c|c|}
\hline BNS No. & Symbol & Litvin No. & Page & Notes \\
\hline 63.463 & $C m c^{\prime} m^{\prime}$ & 63.7 .517 & 1570 & $(12)$ \\
\hline 63.464 & $C m^{\prime} \mathrm{cm}^{\prime}$ & 63.8 .518 & 1572 & (12) \\
\hline 63.465 & $C m^{\prime} c^{\prime} m^{\prime}$ & 63.9 .519 & 1574 & (12) \\
\hline 64.469 & Cmca & 64.1 .528 & 1592 & \\
\hline 64.470 & Cmca1' & 64.2 .529 & 1594 & \\
\hline 64.471 & $\mathrm{Cm}^{\prime} \mathrm{ca}$ & 64.3 .530 & 1596 & \\
\hline 64.472 & $C m c^{\prime} a$ & 64.4 .531 & 1598 & \\
\hline 64.473 & Cmca' & 64.5 .532 & 1600 & \\
\hline 64.474 & $C m^{\prime} c^{\prime} a$ & 64.6 .533 & 1602 & (13) \\
\hline 64.475 & $C m c^{\prime} a^{\prime}$ & 64.7 .534 & 1604 & \\
\hline 64.476 & $C m^{\prime} c a^{\prime}$ & 64.8 .535 & 1606 & \\
\hline 64.477 & $C m^{\prime} c^{\prime} a^{\prime}$ & 64.9 .536 & 1608 & \\
\hline 65.481 & Cmmm & 65.1 .545 & 1626 & (14) \\
\hline 65.482 & $C m m m 1^{\prime}$ & 65.2 .546 & 1628 & (14) \\
\hline 65.483 & $\mathrm{Cm}^{\prime} \mathrm{mm}$ & 65.3 .547 & 1630 & (12) \\
\hline 65.484 & $\mathrm{Cmmm}^{\prime}$ & 65.4 .548 & 1632 & (12) \\
\hline 65.485 & $C m^{\prime} m^{\prime} m$ & 65.5 .549 & 1634 & (12) \\
\hline 65.486 & $C m m^{\prime} m^{\prime}$ & 65.6 .550 & 1636 & (12) \\
\hline 65.487 & $C m^{\prime} m^{\prime} m^{\prime}$ & 65.7 .551 & 1638 & $(12),(15)$ \\
\hline 66.491 & Cccm & 66.1 .564 & 1664 & (14) \\
\hline 66.492 & Cccm $1^{\prime}$ & 66.2 .565 & 1666 & (14) \\
\hline 66.493 & $C c^{\prime} \mathrm{cm}$ & 66.3 .566 & 1668 & (12) \\
\hline 66.494 & $\mathrm{Cccm}^{\prime}$ & 66.4 .567 & 1670 & (12) \\
\hline 66.495 & $C c^{\prime} c^{\prime} m$ & 66.5 .568 & 1672 & (12) \\
\hline 66.496 & $C c c^{\prime} m^{\prime}$ & 66.6 .569 & 1674 & (12) \\
\hline 66.497 & $C c^{\prime} c^{\prime} m^{\prime}$ & 66.7 .570 & 1676 & (12) \\
\hline 67.501 & Cmma & 67.1.577 & 1690 & \\
\hline 67.502 & Cmma1' & 67.2 .678 & 1692 & \\
\hline 67.503 & $C m^{\prime} m a$ & 67.3.579 & 1694 & (16) \\
\hline 67.504 & $C m m a^{\prime}$ & 67.4 .580 & 1696 & \\
\hline 67.505 & $C m^{\prime} m^{\prime} a$ & 67.5 .581 & 1698 & (17) \\
\hline 67.506 & $C m m^{\prime} a^{\prime}$ & 67.6.582 & 1700 & \\
\hline 67.507 & $C m^{\prime} m^{\prime} a^{\prime}$ & 67.7 .583 & 1702 & \\
\hline 68.511 & Ccca & 68.1 .594 & 1724 & \\
\hline 68.512 & Ccca $1^{\prime}$ & 68.2 .595 & 1726 & \\
\hline 68.513 & $C c^{\prime} c a$ & 68.3 .596 & 1728 & \\
\hline 68.514 & $\mathrm{Ccca}^{\prime}$ & 68.4 .597 & 1730 & \\
\hline 68.515 & $C c^{\prime} c^{\prime} a$ & 68.5.598 & 1732 & \\
\hline 68.516 & $C c c^{\prime} a^{\prime}$ & 68.6 .599 & 1734 & (18) \\
\hline 68.517 & $C c^{\prime} c^{\prime} a^{\prime}$ & 68.7 .600 & 1736 & \\
\hline 69.521 & Fmmm & 69.1 .605 & 1746 & $(19),(20)$ \\
\hline 69.522 & $F m m m 1^{\prime}$ & 69.2 .606 & 1748 & $(19),(20)$ \\
\hline 69.523 & $F m^{\prime} m m$ & 69.3.607 & 1751 & $(19),(20)$ \\
\hline 69.524 & $F m^{\prime} m^{\prime} m$ & 69.4.608 & 1753 & $(19),(20)$ \\
\hline 69.525 & $F m^{\prime} m^{\prime} m^{\prime}$ & 69.5 .609 & 1755 & \\
\hline 70.527 & $F d d d$ & 70.1 .616 & 1769 & \\
\hline 70.528 & $F d d d 1^{\prime}$ & 70.2 .617 & 1771 & \\
\hline 70.529 & $F d^{\prime} d d$ & 70.3 .618 & 1774 & \\
\hline 70.530 & $F d^{\prime} d^{\prime} d$ & 70.4 .619 & 1776 & \\
\hline 70.531 & $F d^{\prime} d^{\prime} d^{\prime}$ & 70.5 .620 & 1778 & \\
\hline 71.533 & Immm & 71.1.621 & 1780 & \\
\hline 71.534 & $\operatorname{Immm} 1^{\prime}$ & 71.2 .622 & 1782 & \\
\hline 71.535 & $\mathrm{Im}^{\prime} \mathrm{mm}$ & 71.3.623 & 1784 & \\
\hline 71.536 & $I m^{\prime} m^{\prime} m$ & 71.4.624 & 1786 & \\
\hline 71.537 & $I m^{\prime} m^{\prime} m^{\prime}$ & 71.5 .625 & 1788 & \\
\hline 72.539 & Ibam & 72.1 .630 & 1798 & \\
\hline 72.540 & $\operatorname{Ibam} 1^{\prime}$ & 72.2 .631 & 1800 & \\
\hline 72.541 & $I b^{\prime} a m$ & 72.3 .632 & 1802 & \\
\hline 72.542 & Ibam' $^{\prime}$ & 72.4 .633 & 1804 & \\
\hline 72.543 & $I b^{\prime} a^{\prime} m$ & 72.5 .634 & 1806 & \\
\hline 72.544 & $I b a^{\prime} m^{\prime}$ & 72.6 .635 & 1808 & \\
\hline 72.545 & $I b^{\prime} a^{\prime} m^{\prime}$ & 72.7 .636 & 1810 & \\
\hline 73.548 & Ibca & 73.1 .643 & 1824 & \\
\hline
\end{tabular}

Table 5 (continued)

\begin{tabular}{lllll}
\hline BNS No. & Symbol & Litvin No. & Page & Notes \\
\hline 73.549 & $I b c a 1^{\prime}$ & 73.2 .644 & 1826 & \\
73.550 & $I b^{\prime} c a$ & 73.3 .645 & 1828 & \\
73.551 & $I b^{\prime} c^{\prime} a$ & 73.4 .646 & 1830 & \\
73.552 & $I b^{\prime} c^{\prime} a^{\prime}$ & 73.5 .647 & 1832 & \\
\hline 74.554 & $I m m a$ & 74.1 .650 & 1838 & \\
74.555 & $I m m a 1^{\prime}$ & 74.2 .651 & 1840 & \\
74.556 & $I m^{\prime} m a$ & 74.3 .652 & 1842 & \\
74.557 & $I m m a^{\prime}$ & 74.4 .653 & 1844 & \\
74.558 & $I m^{\prime} m^{\prime} a$ & 74.5 .654 & 1846 & \\
74.559 & $I m m^{\prime} a^{\prime}$ & 74.6 .655 & 1848 & \\
74.560 & $I m^{\prime} m^{\prime} a^{\prime}$ & 74.7 .656 & 1850 & \\
\hline
\end{tabular}

Notes. (1) Koptsik (1966): glide planes in dir1 should be red; (2) Koptsik (1966): one glide plane in dir2 missing; (3) Koptsik (1966): there should be no black glide planes in dir1; (4) Koptsik (1966): one red arrow missing; (5) Koptsik (1966): there should be no mirror planes in dir2; (6) Litvin (2008)-SM: rotations in dir1 should be black, screws in dir2 should be red; (7) Litvin (2008)-SM: rotations in dir1 should be red, screws in dir2 should be black; (8) Litvin (2008)-SM, dir3: mirror plane should be red; (9) Koptsik (1966), dir3: red screws missing; (10) Litvin (2008)-SM, dir2: screws should be red; (11) Koptsik (1966), dir3: one screw axis missing; (12) Litvin (2008)-SM \& Koptsik (1966): omit $n$-glide in dir3; (13) Litvin (2008)-SM: mirror and glide planes in dir1 should be red; (14) Litvin (2008)-SM: omit $n$-glide in dir3; (15) Koptsik (1966): inversion points should be red; (16) Litvin (2008)-SM: inversion points should be red; (17) Litvin (2008)-SM, dir2: mirror and glide planes should be red; (18) Koptsik (1966), dir2: some red glide planes missing; (19) Litvin (2008)-SM: omit $n$-glides in dir1, dir2 and dir3; (20) replace axial planes by $e$-glide in $\operatorname{dir} 1$ and $\operatorname{dir} 2$.

\section{Shubnikov space groups related to point group $\mathbf{m m 2}$}

Table 2 lists the orthorhombic BW2 space-group types with rotations or screws in one symmetry direction, and mirror or glide planes perpendicular to the two other symmetry directions. Horizontal lines separate types belonging to different BNS superfamilies.

Notice that if the centring part of the OG symbol is $P_{2 a}, A_{2 a}$ or $A_{I}$, then a factor $1 / 2$ appears in column 'dir1'; if the centring part of the OG symbol is $P_{2 b}$ then a factor $1 / 2$ appears in 'dir2'; and if the centring part of the OG symbol is $P_{2 c}, C_{2 c}$ or $C_{I}$, then a factor $1 / 2$ appears in 'dir3'. If the centring part of the $\mathrm{OG}$ symbol is $P_{A}$, then a factor $1 / 2$ appears in 'dir2' and 'dir3'; if the centring part of the OG symbol is $P_{C}$, then a factor $1 / 2$ appears in 'dir1' and 'dir2'; and if the centring part of the OG symbol is $P_{F}$, then a factor $1 / 2$ appears in 'dir1', 'dir2' and 'dir3'. Remember that a factor $1 / 2$ in 'dir3' leads to doubling of the height values. These rules also hold for Tables 1 and 4 .

The last column contains remarks and corrections to the DSEs in Litvin (2008)-SM and in Koptsik (1966). In both cases, dir1 tells us that a rotation or screw axis and the normal to a mirror or glide plane is vertical, dir2 tells us that it is horizontal, and dir3 tells us that it is perpendicular to the plane of the drawing.

The majority of remarks are connected with glide planes. A primed (unprimed) symmetry glide may be considered as a primed (unprimed) mirror reflection followed by an unprimed translation $\mathbf{t}$. Whereas the combined operation belongs to the Shubnikov space group $\mathbf{S}$ under consideration, the mirror reflection alone and $\mathbf{t}$ alone are not elements of $\mathbf{S}$. The glide vector $\mathbf{t}$ has the property that $2 \mathbf{t}$ is (and $\mathbf{t}$ is not) an unprimed symmetry translation. As an example for the orthorhombic lattice with conventional cell spanned by vectors $\mathbf{a}, \mathbf{b}$ and $\mathbf{c}$, we 
consider a symmetry glide plane $\perp \mathbf{a}$. If the lattice is neither $A$ nor $F$-centred, then $\mathbf{t}$ will have the form $\mathbf{t}=\frac{1}{2}(h \mathbf{b}+k \mathbf{c})$, where $h$ and $k$ are integers that are not both even. The glide vector of a symmetry glide with a plane in a fixed orientation and position is determined only up to an unprimed (symmetry) translation. Therefore we may choose $\mathbf{t}=\mathbf{b} / 2$ if $h$ is odd and $k$ is even, $\mathbf{t}=$ $\mathbf{c} / 2$ if $h$ is even and $k$ is odd, or $\mathbf{t}=\frac{1}{2}(\mathbf{b}+\mathbf{c})$ if $h$ and $k$ are odd. If the lattice is $A$ - or $F$-centred, then $\frac{1}{2}(\mathbf{b}+\mathbf{c})$ is an unprimed (symmetry) translation, i.e. a mirror reflection followed by a translation $\frac{1}{2}(\mathbf{b}+\mathbf{c})$ becomes equivalent to the mirror reflection alone; a mirror reflection followed by a translation $\mathbf{b} / 2$ becomes equivalent to the mirror reflection followed by a translation $\mathbf{c} / 2$. The latter case is referred to as a 'double glide' or ' $e$-glide'. The $e$-glides are denoted by a horizontal and a vertical arrow with common origin if the glide plane is parallel to the projection plane. If the glide plane is normal to the projection plane, they were denoted either as a dashed or dotted line in ITXC52 and in Koptsik (1966), as was usual before the introduction of the dash-dot-dot line in ITC95. The $e$-glides are denoted both by a dashed and a dotted line in Litvin (2008)-SM; the mirrors often both as mirror and as $n$-glide in Litvin (2008)-SM and Koptsik (1966). Representing equivalent symmetry elements by two symbols is both confusing and unnecessarily complicated, and it forced Litvin (2008)-SM to distribute the symmetry elements over two diagrams in some cases.

Table 3 lists the orthorhombic monochrome, grey and BW1 space-group types with rotations or screws in one symmetry direction, and mirror or glide planes perpendicular to the two other symmetry directions. Horizontal lines separate types belonging to different BNS superfamilies.

\section{Orthorhombic Shubnikov space groups related to point group $\mathbf{m m m}$}

Table 4 lists the orthorhombic BW2 space-group types with mirror or glide planes perpendicular to all three symmetry directions. Horizontal lines separate types belonging to different BNS superfamilies.

The remarks in Table 4 to the diagram of symmetry elements given by Litvin (2008)-SM for 63.467 are illustrated in Fig. 1.

Table 5 lists the orthorhombic monochrome, grey and BW1 space-group types with mirror or glide planes perpendicular to all three symmetry directions. Horizontal lines separate types belonging to different BNS superfamilies.

\section{Conclusions}

Comparison of the diagrams of symmetry elements (DSEs) for the orthorhombic point groups given in Koptsik (1966) and in Litvin (2008)-SM showed the following main results.

In Koptsik (1966) some of the symmetry elements that differ only by a shift are missing in several cases. Lines deli- miting the projection of the conventional cell appear as mirror planes in several cases. Mirror planes parallel to the plane of the diagram also appear sometimes as $n$-glides in the case of $C$-centred cells. In particular, in the case of vertical lines, it is sometimes not clear whether they denote mirrors, axial or diagonal glides.

In Litvin (2008)-SM $e$-glides are treated as two independent axial glides, which is misleading and unnecessarily complicates the DSEs. Mirror planes also sometimes appear as $n$-glides in the case of $C$ - and $A$-centred cells.

Notice that if the lattice part of the OG symbol is $P_{F}\left(P_{A}\right.$ or $\left.P_{C}\right)$ then only $\frac{1}{4}\left(\frac{1}{2}\right)$ of the symmetry operations are listed under the heading 'Symmetry Operations' in Litvin (2008)-SM.

The standard symbols for BW2-type space groups proposed by Opechowski \& Guccione (1965) and by Belov et al. $(1957 a, b)$, respectively, often correspond to different orientations of the space group, as shown in columns dir1, dir2 and dir3 of Tables 1, 2 and 4.

Whereas Litvin (2001) needs 216 pages of supplementary material to describe the meaning of the OG symbols, the meaning of the BNS symbols is obvious, including those for the BW2 types. Therefore the BNS symbols should be recognized as standard.

The author is grateful to Professor D. B. Litvin for discussions which gave him a clearer understanding of the definition of the Opechowski-Guccione symbols for BW2-type space groups.

\section{References}

Belov, N. V., Neronova, N. N. \& Smirnova, T. S. (1957a). Kristallografiya, 2, 315-325.

Belov, N. V., Neronova, N. N. \& Smirnova, T. S. (1957b). Sov. Phys. Crystallogr. 2, 311-322.

Borovik-Romanov, A. S. \& Grimmer, H. (2003). International Tables for Crystallography, Vol. D, edited by A. Authier, pp. 105-149. Dordrecht: Kluwer Academic Publishers.

International Tables for Crystallography (1983). Vol. A, Space-Group Symmetry, 1st ed., edited by Th. Hahn. Dordrecht: Reidel Publishing Company.

International Tables for Crystallography (1995). Vol. A, Space-Group Symmetry, 4th ed., edited by Th. Hahn. Dordrecht: Kluwer Academic Publishers.

International Tables for X-ray Crystallography (1952). Vol. I, Symmetry Groups, edited by N. F. M. Henry \& K. Lonsdale. Birmingham: Kynoch Press.

Koptsik, V. A. (1966). Shubnikov Groups. Moscow: Izd. M. U.

Litvin, D. B. (1998). Acta Cryst. A54, 257-261.

Litvin, D. B. (2001). Acta Cryst. A57, 729-730.

Litvin, D. B. (2008). Acta Cryst. A64, 419-424.

Opechowski, W. (1986). Crystallographic and Metacrystallographic Groups. Amsterdam: North-Holland.

Opechowski, W. \& Guccione, R. (1965). Magnetism, edited by G. T. Rado \& H. Suhl, Vol. II, Part A, pp. 105-165. New York, London: Academic Press. 